\title{
High Performance Direction Finding Algorithm Based on Phase Locked Loop
}

\author{
Han Trong Thanh, Vu Van Yem \\ School of Electronics and Telecommunications, Hanoi University of Science and Technology \\ No. 1, Dai Co Viet Road, Hai Ba Trung District, Hanoi, Vietnam
}

Correspondence: Han Trong Thanh, thanh.hantrong@hust.edu.vn

Manuscript communication: received 12 March 14, accepted 20 October 2014

\begin{abstract}
Using Phase Locked Loop based single channel Direction Finding (DF) system to estimate the bearing angle or the coordinates of an incoming radio signal(s) has much more advantages than multiple receiver system does in many practical scenarios such as mobile communication. This method utilizes a bank of Phase Locked Loops (PLLs) to calculate the differential phase of signal received by an M-element uniform circular antenna array with a commutative switch followed by single channel Software Defined Radio (SDR) receiver. One important factor when using conventional phase locked loop is the requirement of the small convergence rate of the algorithm compared to the switching cycle. In order to achieve small convergence rate, we propose a method for DOA estimation with low computation complexity that improves significantly the performance of conventional PLLs system. An analysis of the challenges of computation complexity in this algorithm is presented. The simulation results for DOA estimation using the proposed structure with low complexity are shown to verify the performance of the system.
\end{abstract}

Keywords- Direction Finding (DF), Direction of Arrival (DOA), Software Defined Radio (SDR), Digital PLL.

\section{INTRODUCTION}

Radio Direction Finding (DF) is a technique that identifies the bearing angle or the coordinates of an incoming radio signals. The most important information that estimated by a DF system is the Direction of Arrival (DOA). DF systems have many applications in Radio Navigation, Emergency Aid and intelligent operations, etc... DF system can be classified by many ways. Based on the system architecture, we have two types: single channel DF and multi-channel DF. Multichannel DF system refers to the use of an antenna array with multiple radio receivers in parallel. Multi-channel DF system provides quite accurate DOA estimation at the expenses of weight, computation complexity and hardware cost. Because of this reason, the deployment of multi-channel is impractical in some scenarios such as mobile communication. On the other hand, Singlechannel DF refers to the use of a multi-antennas array connected to a single channel radio receiver by a radio frequency switch. The approach using DF obviously offers some benefits such as mobility and lower power consumptions. However, due to using switching cycle, it cannot look at each antenna simultaneously, which would be the case if one were to use multiple receivers, also known as multi-channel DF. Therefore more complex operations are needed at the antenna in order to present the signal to the receiver. Based on the signal processing manner, the two main categories that a single channel DF algorithm falls into are amplitude comparison and phase comparison [1, 2]. Some algorithms can be hybrids of the two.
In [3-6], a single channel phase comparison DF algorithm known as the Phase Locked Loop algorithm is introduced. According to the results obtained, it is well-performed in the laboratory environment. This method utilizes a bank of Phase Locked Loops (PLLs) to calculate the differential phase of signal received by an M-element uniform circular antenna array with a commutative switch followed by single channel Software Defined Radio (SDR) receiver. Similar to other phase based methods, the challenge when using PLLs is computation's complexity. In fact, one important factor when using phase locked loop is the requirement of small convergence rate of the algorithm compared to the switching cycle. The more complex the computation is, the more time is needed. When the source of signal is mostly static, the problem is inconsiderable. However, if the target moves at high speed, the DOA changes rapidly. Thus, it is important to reduce the time of DOA estimation. Moreover, the computation complexity has a large impact on the performance of any radio receiver. That is why we need to have a simpler complex in computation of DOA information.

In this paper, we investigate a low complexity DF algorithm based on digital PLL. We model the DF system, including of an eight element circular antenna array connected to a single channel software defined radio receiver by a digital 8-to-1 switching circuit which switches sequentially around the elements. The time divided signals are then in turn leaded to one of eight PLLs which then track the phase of the signal at each antenna. The tracked phases are then differentiated and fed into a signal processing block to produce DOA 


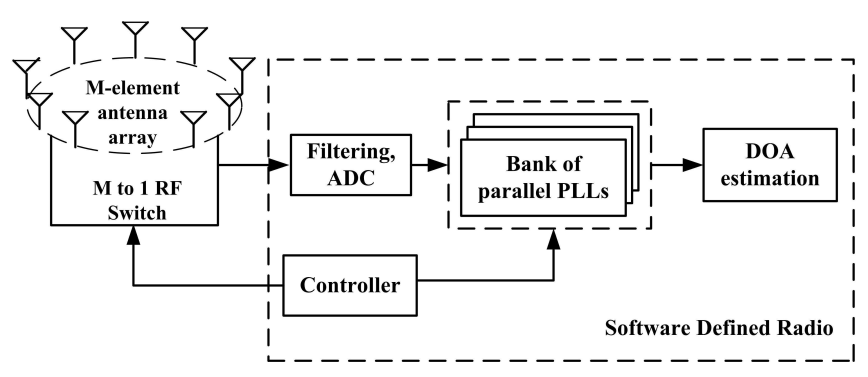

Figure 1. PLL DF system block diagram block

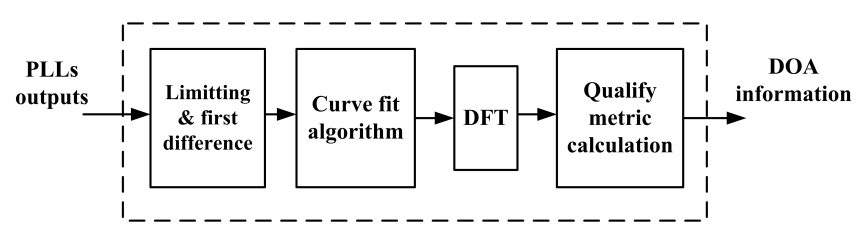

Figure 2. DOA estimation processing block

estimation. In this block, the PLL-DOA algorithm is presented first. Then, we execute our signal processing method with the same input. By this way, we can assess the efficiency of the proposed algorithm in comparison with the original algorithm as well as the performance of the DF system.

The paper is organized as follows. Section 2 describes the system architecture and the model of the incoming signals. Section 3 presents in detail the PLL-DOA algorithm with low computation complexity. In Section 4, we examine this algorithm for multiple signal sources. The simulation results are presented in the Section 5 . The conclusion is given in the section 6 .

\section{System Architecture and Signal Model}

\subsection{System Architecture}

The DF system based on digital PLLs, as shown in Figure 1, involves an M-element uniform circular antenna with an $M$-to-1 RF switching connected to a software defined platform receiver. In general, the interspacing between antenna's elements of an M element array is less than half of wavelength of the highest frequency is used. In our research, we employed an 8 element array with the radius equals half of the interested wavelength for practical implementation purpose. The receiver will filter out and convert the RF signal to a complex base-band signal. This signal is then fed into one of 8 paralleled PLLs which is used to track the phase of the incoming signal at each element. Figure 2 shows the block diagram of the processing flow after the PLLs.

After being tracked, the phase at each element will be differentiated to remove any phase offset and data's amplitude. As a result, a phase difference metric is created between adjacent antenna elements. These phase difference estimates are called "the given difference curves". Because of the known location of each antenna element, a set of theoretical phase differences can be created. This set of curves is called "the target difference curves". The mean square error (MSE) is calculated

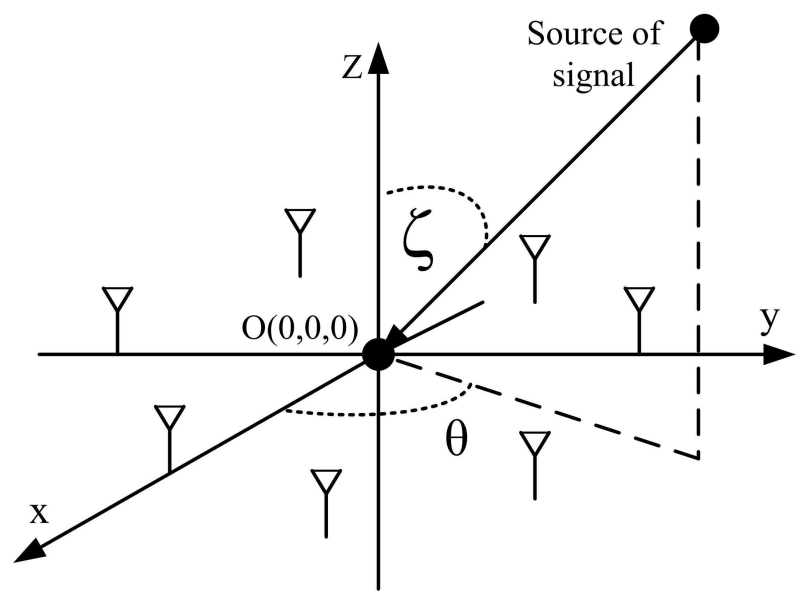

Figure 3. Antenna array in the coordinate system

between the given and target difference curves. This comparison is called "the Curve fit algorithm". The Curve fit algorithm removes any ambiguities on the PLL data related to the modulation on the signal. DFT is then performed on the PLL data to get the DOA information and produce a qualify metric to estimate DOA.

\subsection{Signal Model}

In our research, we have defined the reference point as the origin of the three Dimensional Cartesian coordinate system shown in Figure 3. The phase difference between any antenna and the reference point is given below:

$$
\Delta \Psi=\beta\left(x_{m} \cos \theta \sin 1+y_{m} \sin \theta \sin 1+z_{m} \cos \zeta\right),
$$

where $x_{m}, y_{m}, z_{m}$ are the three dimensional coordinates of $m^{\text {th }}$ antenna element, $\beta=\frac{2 \pi}{\lambda}$ is the propagation factor and $\zeta$ and $\theta$ are the DOA information in elevation and azimuth, respectively.

The phase at the $m^{\text {th }}$ antenna relative to the reference point is given as

$$
a_{m}=g_{m} e^{-j \Delta \Psi},
$$

where $g_{m}$ is the gain of the $m^{\text {th }}$ antenna element.

The baseband output signal at the $m^{\text {th }}$ antenna is:

$$
x_{m}(t)=s(t) a_{m},
$$

where $s(t)$ is the incoming signal. This work concerns about the signal in the same plane with antenna array. This means that we estimate the DOA of signal of interest in azimuth, $\zeta=90^{\circ}, z_{m}=0$.

In an $M$ element circular antenna array with a radius $r$,

$$
x_{m}=r \cos \left(\frac{2 \pi \mathrm{m}}{M}\right), y_{m}=r \sin \left(\frac{2 \pi \mathrm{m}}{M}\right)
$$

so

$$
a_{m}=g_{m} \cdot e^{-j \frac{2 \pi r}{\lambda} \cos \left(\frac{2 \pi m}{M}-\theta\right)}
$$

and

$$
x_{m}(t)=s(t) g_{m} e^{-j \frac{2 \pi r}{\lambda} \cos \left(\frac{2 \pi m}{M}-\theta\right)}
$$


Due to the modulation $s(t)=c(t) \cdot e^{-j \varphi}$, where $c(t)$ is data related to modulation and $\varphi$ is the phase offset, the signal received can be written as follows:

$$
x_{m}(t)=c(t) \cdot g_{m} \cdot e^{-j\left(\frac{2 \pi r}{\lambda} \cos \left(\frac{2 \pi m}{M}-\theta\right)+\varphi\right)}
$$

In the Equation (7), we can see that the $c(t)$ is the amplitude and the exponential component is the phase of incoming signal at each antenna element that will be tracked by $m^{\text {th }}$ PLL.

\section{High Performance PlL-DOA Algorithm}

\subsection{PLL-DOA Algorithm}

According to Equation (7), if we do not consider about the data of modulation, the output of the $m^{\text {th }}$ PLL is:

$$
\phi_{m}=\frac{2 \pi \mathrm{r}}{\lambda} \cos \left(\frac{2 \pi \mathrm{m}}{M}-\theta\right)+\varphi
$$

$(M=8$ in our model). From which, we can calculate the DOA, $\theta$, by the following equation:

$$
\theta=\arccos \left(\frac{\phi_{m}-\varphi}{A}\right)-\frac{2 \pi \mathrm{m}}{M}
$$

where $A=\frac{2 \pi \mathrm{r}}{\lambda}=\pi$ (due to the design). However, because of the data modulation, the phase tracked is:

$$
\begin{gathered}
\phi_{m}^{\prime}=\frac{2 \pi \mathrm{r}}{\lambda} \cos \left(\frac{2 \pi \mathrm{m}}{M}-\theta\right)+\varphi+n_{m} \pi, \\
(m=0,1, \ldots, M-1)
\end{gathered}
$$

where $\mathrm{n}$ is factor of modulation (i.e. $n=0, \pm 1$ in case the modulation type is BPSK). It shows the incorrect result for DOA if Equation (8) is used. In order to estimate the DOA, we must know $n_{m}$ first. If we try to find the $n_{m}$ sequence now, there will be $(2 N-1)^{M}$ possibilities ( $\mathrm{N}$ is the modulation level). In addition, we do not know the constant phase offset $\varphi$. Besides all of these difficulties, we also have to consider the PLLs only track the phases between $[-\pi, \pi]$. If the real phase of the signal at each antenna is outside of range $-\pi$ to $\pi$, the PLLs will map the phase back by subtracting or adding the phase with $2 \pi$. Thus, the phases coming from PLLs and the target phases can differ by $2 \pi$. This point is illustrated in Figure 4. The "theoretical phases" are phases without phase offsets. The "expected phases" line presents the phase measured at each antenna element. The "actual phases" are outputs of PLLs. At $2^{\text {nd }}$, $3^{\text {rd }}$ and $8^{\text {th }}$ antennas, the phases of signal do not lie in the range $[-\pi, \pi]$. Thus, the PLLs take them back.

Because of above analysis, we can not immediately get the true DOA by the recorded phases from the PLLs. To overcome this problem, we should remove all ambiguities by the steps as follows.

After being tracked, the phases will be differentiated to remove the phase offset occurring in all phases. We consider "the first target difference curve" as

$$
\begin{aligned}
\Delta \phi_{m} & =\phi_{m}-\phi_{m-1} \\
& =-2 A \sin \left(\frac{\pi}{M}\right) \sin \left(\frac{2 \pi \mathrm{m}}{M}-\frac{\pi}{M}+\theta\right)
\end{aligned}
$$

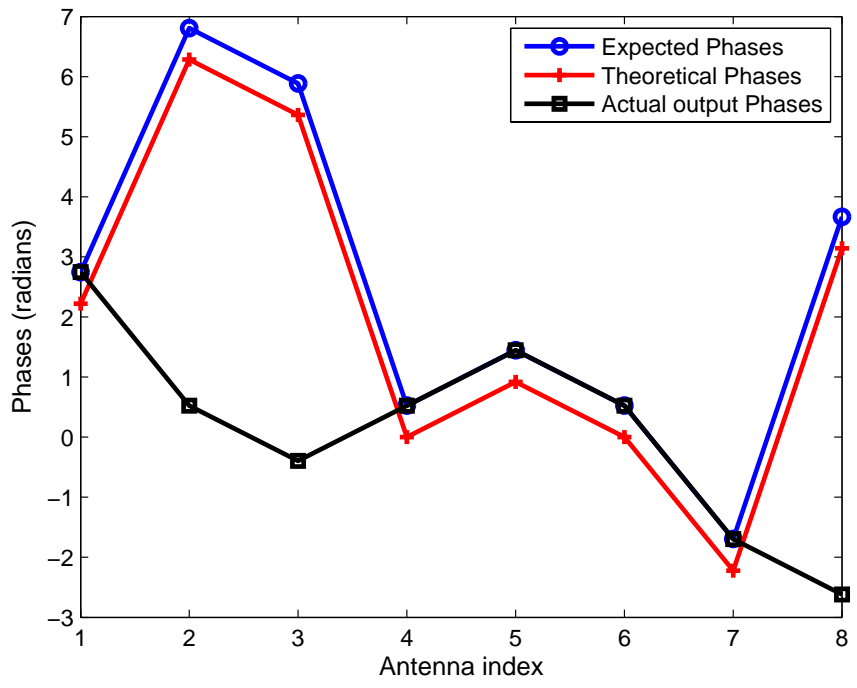

Figure 4. Theoretical and PLL output phase at each antenna.

In case of the data modulation, "the first given difference curve" is given as

$$
\begin{aligned}
\Delta \phi_{m}^{\prime} & =\phi_{m}^{\prime}-\phi_{m-1}^{\prime} \\
& =-2 A \sin \left(\frac{\pi}{M}\right) \sin \left(\frac{2 \pi \mathrm{m}}{M}-\frac{\pi}{M}+\theta\right)+\Delta n_{m} \pi
\end{aligned}
$$

Our objective is to find the $\Delta n_{m}$. Although the difference $\Delta n_{m}$ has much larger range than $n_{m}$, this sequence is still found by the following way.

The target curve is aimed to be found from the given curve. To do that, we have to create the target difference curves database. If we have $\mathrm{N}$ level modulation, we have $(2 N-1)$ values of difference phase. We have $\mathrm{M}$ antenna elements so we have $(2 N-1)^{M}$ sequences. However, by using the maximum value $\Delta \phi_{m}, D_{\max }$, we can eliminate several of these possibilities. From Equation (11), $D_{\max }=2 A \sin \left(\frac{\pi}{M}\right)$ with $A=\pi, M=8$ so $D_{\max }<\pi$. Therefore, any points at "the first target difference curve" cannot exceed $[-\pi, \pi]$. Because the algorithm tries to get the target curve from the given curve, we can eliminate several possibilities that makes the first difference exceeds $[-\pi, \pi]$. It means that for BPSK modulation, we have only two phase difference values: $0, \pi$.

For $M=8, \Delta n_{m}=(0,1)$, we have $2^{8}=256$ possible curves. We create a table to show all of these possibilities. Next, we divide the possible DOA, $\theta$, with range in $[-\pi, \pi]$ radian into N1 bins, and create the target first difference curve for each possible DOA. We then select one of the $N_{1} \times 256$ first given difference curves and compare to the first target difference curves, i.e. choose the one for which the squared error is minimized. Once we have found the sequence $\Delta n_{m}$, we can use the first difference curve to calculate the DOA.

After removing the $\Delta n_{m}$, the target difference curve 


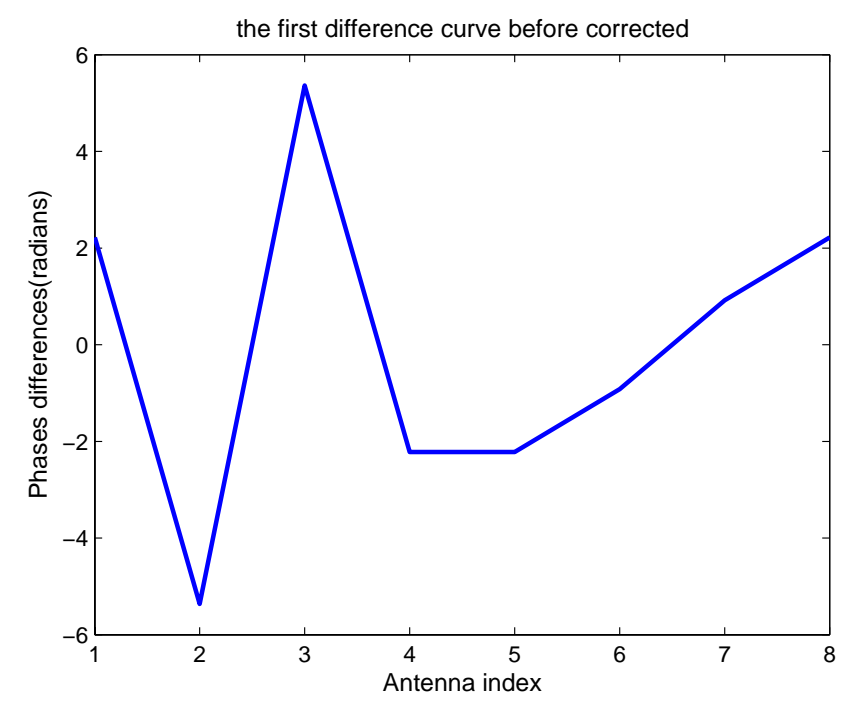

Figure 5. The first phase difference curve before corrected without modulation.

can be represented as following vector:

$$
f[m]=\left[\begin{array}{c}
-2 A \sin \left(\frac{\pi}{M}\right) \sin \left(-\frac{\pi}{M}+\theta\right) \\
-2 A \sin \left(\frac{\pi}{M}\right) \sin \left(\frac{2 \pi}{M}-\frac{\pi}{M}+\theta\right) \\
\cdots \\
-2 A \sin \left(\frac{\pi}{M}\right) \sin \left(\frac{2 \pi m}{M}-\frac{\pi}{M}+\theta\right)
\end{array}\right]
$$

The FFT of this vector is

$$
F[k]=\sum_{m=0}^{M-1} f[m] e^{\frac{-2 \pi m k}{M}}
$$

All of $F[k]$ are zeros except

$$
F[1]=c\left[\cos \left(\frac{\pi}{M}+\theta\right)+j \sin \left(\frac{\pi}{M}+\theta\right)\right]
$$

Thus the estimated DOA is

$$
\theta=\frac{\pi}{2}-\frac{\pi}{M}-\arg (F[1])
$$

Evidently, the size of the first target difference curve database is still too large. This fact requires a significant number of complex calculations as well as much more time needed for computation.

\subsection{PLL-DOA Algorithm with Low Computation Complexity}

In order to realize the real system, we have to reduce the complexity as well as decrease the calculation time so we propose a new method as follows.

3.2.1 In Case of No Modulation Incoming Signal: When we do not concern the data modulation, the phase of the incoming signal at each antenna element is modeled as Equation (8). By plotting these phases, we can get the sinusoid form as shown in Figure 6. According to the Equation (11), it is clear that the first difference is still a sinusoid. However, due to the impact of PLLs's boundary, the first difference curve does not

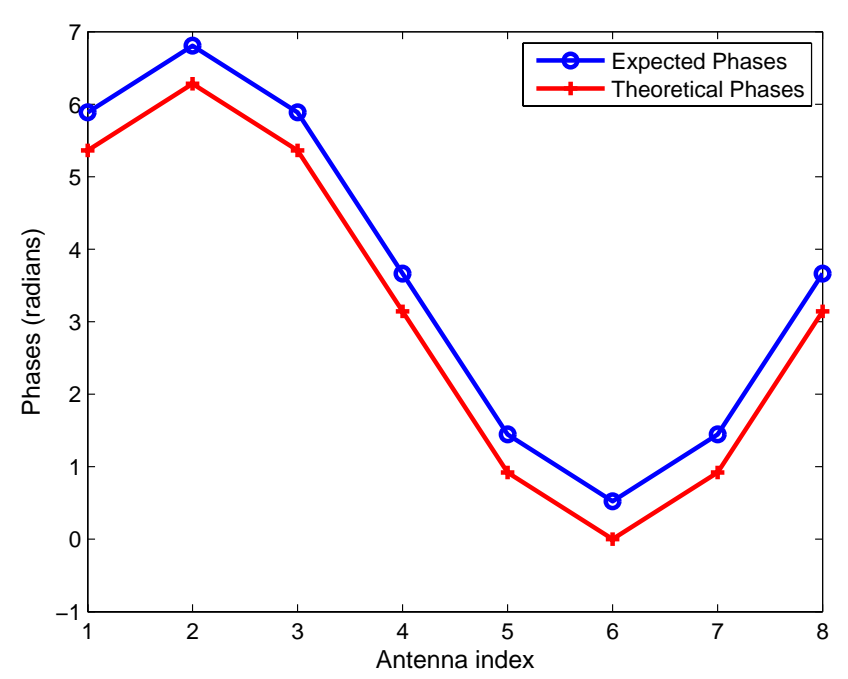

Figure 6. Theoretical phase at each antenna element in case of no modulation.

match a sinusoid as seen in Figure 5 though there is nomodulation. In order to solve this problem, we calculate "the second target difference curve":

$$
\begin{aligned}
\Delta \phi_{m}^{\prime \prime} & =\Delta \phi_{m}-\Delta \phi_{m-1} \\
& =\frac{-8 \pi \mathrm{r}}{\lambda} \sin ^{2}\left(\frac{\pi}{M}\right) \cos \left(\frac{2 \pi \mathrm{m}}{M}-\frac{2 \pi}{M}+\theta\right)
\end{aligned}
$$

The purpose of the second difference curve is to locate the antenna element at which the phase is added or subtracted by $2 \pi$. For each peak in "the first difference given curve", we compare with the corresponding points in "the second difference given curve". If the consecutive values exceed $\pi$ or $-\pi$ then we subtract or add $2 \pi$ to the first difference. In Figure 5, we can see the peaks at $2^{\text {nd }}$ and $3^{\text {rd }}$ antennas in the first difference curve. Now we investigate how the second difference corrects the first difference. The second difference curve is presented in Figure 7.

In the second difference curve, there have two consecutive couple of values exceed the range of $-\pi$ to $\pi$. They are $\left(2^{\text {nd }}, 3^{\text {rd }}\right)$ and $\left(3^{\text {rd }}, 4^{\text {th }}\right)$ corresponding to the peaks at $2^{\text {nd }}$ and $3^{\text {rd }}$ antennas in the first difference curve. So we add phase at antenna $2^{\text {nd }}$ by $2 \pi$ and subtract phase at $3^{\text {rd }}$ by $2 \pi$. The first difference curve after being corrected is shown in Figure 8.

After corrected, the target difference curve matches the sinusoid as defined in Equation (11). By using the Equation (13) to Equation (16), we can easily get the DOA information.

\subsubsection{In Case of Modulation Incoming Signal:}

Similar to the original algorithm, we also have to calculate "the first target difference curve" and "the first given difference curve" in succession. In case of $N$ level modulation and $M$ antenna elements, we have the number of possible curves as:

$$
a=(2 N-1)^{M}
$$

If we use the original algorithm, we can eliminate a lot of these possibilities. However, the number of pos- 


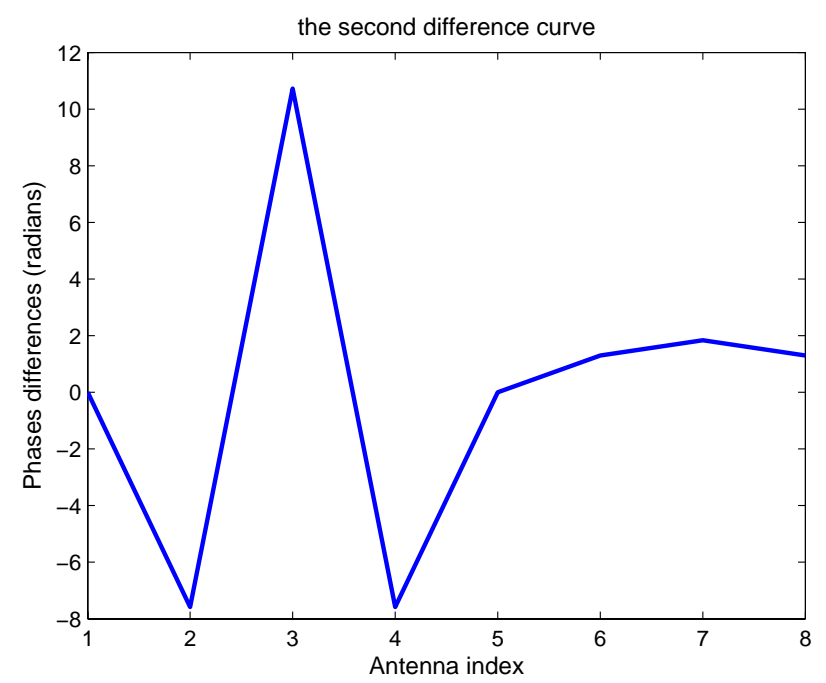

Figure 7. The second phase difference (in case of no modulation).

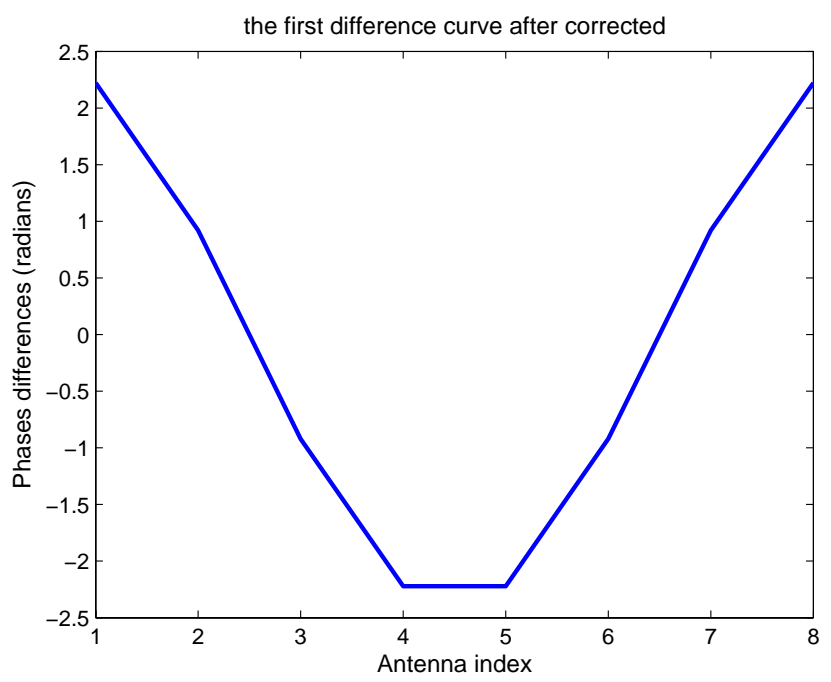

Figure 8. The first phase difference after corrected.

sible curves is still an exponential function of M. Our objective is to reduce the complexity of computation. Instead of having to compute and find in $(2 N-1)^{M}$ possibilities, we can reduce to $(2 N-1) \times M$ findings. We do not have to create the database of target curve. The possible target curves will vary for different times. For $\mathrm{N}$ level modulation, we have $(2 N-1)$ phase difference values. From the first to the last antenna element, we assume that one of $(2 N-1)$ different values occur at that element. We use the assumed value of $\Delta n_{m}$ to calculate a value of possible DOA. We consider this DOA as assumed DOA obtained by using Equation (12) in which $\Delta \phi_{m}^{\prime}$ is a point on "the first given difference curve". After that, based on Equation (11), we calculate the possible target difference curve corresponding to the calculated assumed DOA. Then we use the given curve and the possible target curve to estimate $\Delta n_{m} \mathrm{~s}$. The target curve is added by $\Delta n_{m} \mathrm{~s}$ and the squared error of the target curve and the given curve are calculated. We choose the sequence $\Delta n_{m}$ for which the value of squared error is minimized. By this algorithm, the number of possible curves are only

$$
b=(2 N-1) \times M
$$

After finding the correct sequence of $\Delta n_{m}$, the DOA information will be estimated by the same method presented in above cases.

It is easy to see that the complexity is significantly reduced by the proposed algorithm. The number of calculation is multiple of the number of antenna element, $(2 N-1) \times M$, instead of an exponential function of this factor, $(2 N-1)^{M}$, as in the conventional PLL algorithm [3-5]. That is why it can be operated in real time thanks to its low computation complexity.

\section{PLL-DOA Estimation With Multiple SigNAL SOURCES}

In practice, there are several radio signals crossing the antenna array simultaneously. The received signal at each antenna element will be the sum of all arrival radio signals. However, due to the behavior of filters and PLLs, only the signal of interest whose frequency is in the lock range of PLLs will be passed. In the presence of multipath, many incoming signals will have the same frequency. If one signal is the line-of-sight signal, it should be significantly stronger than others because it has propagated over a shortest distance. When one signal is the strongest signal, the PLL will track it. In the special situation, there are at least two incoming signals have the same frequency and amplitude. For this case, PLLs will track all of them. In the rest of our work, we will focus on any approaches to solve this problem.

Let us define two signals impinging on the antenna array as

$$
S_{1, m}(t)=A \cos \left(\omega_{c} t+\frac{2 \pi \mathrm{r}}{\lambda} \cos \left(\frac{2 \pi \mathrm{m}}{M}-\theta_{1}\right)+\varphi_{1}\right)
$$

$$
S_{2, m}(t)=A \cos \left(\omega_{c} t+\frac{2 \pi \mathrm{r}}{\lambda} \cos \left(\frac{2 \pi \mathrm{m}}{M}-\theta_{2}\right)+\varphi_{2}\right)
$$

where $\omega_{c}$ is the carrier frequency; $\varphi_{1}, \varphi_{2}$ are the phase offsets and $\theta_{1}, \theta_{2}$ are the DOAs. The output at each antenna is simply modeled as the sum of two signals

$$
S_{m}(t)=S_{1, m}(t)+S_{2, m}(t)
$$

Based on the formula

$$
\cos (a)+\cos (b)=2 \cos \left(\frac{a+b}{2}\right) \cos \left(\frac{a-b}{2}\right)
$$

where

$$
\begin{aligned}
& a=\omega_{c} t+\frac{2 \pi \mathrm{r}}{\lambda} \cos \left(\frac{2 \pi \mathrm{m}}{M}-\theta_{1}\right)+\varphi_{1} \\
& b=\omega_{c} t+\frac{2 \pi \mathrm{r}}{\lambda} \cos \left(\frac{2 \pi \mathrm{m}}{M}-\theta_{2}\right)+\varphi_{2}
\end{aligned}
$$


we have:

$$
\begin{aligned}
a+b= & 2 \omega_{c} t+\varphi_{1}+\varphi_{2} \\
& +\frac{2 \pi \mathrm{r}}{\lambda}\left[\cos \left(\frac{2 \pi \mathrm{m}}{M}-\theta_{1}\right)+\cos \left(\frac{2 \pi \mathrm{m}}{M}-\theta_{2}\right)\right] \\
= & 2 \omega_{c} t+\varphi_{1}+\varphi_{2} \\
& +\frac{4 \pi \mathrm{r}}{\lambda} \cos \left(\frac{2 \pi \mathrm{m}}{M}-\frac{\theta_{1}+\theta_{2}}{2}\right) \cos \left(\frac{\theta_{1}-\theta_{2}}{2}\right)
\end{aligned}
$$

By doing the same way, the difference should be:

$a-b=\varphi_{1}-\varphi_{2}-\frac{4 \pi \mathrm{r}}{\lambda} \sin \left(\frac{2 \pi \mathrm{m}}{M}-\frac{\theta_{1}+\theta_{2}}{2}\right) \sin \left(\frac{\theta_{1}-\theta_{2}}{2}\right)$

It is easy to recognize that the $(a-b)$ term is not the function of carrier frequency thus it relates to the amplitude part of the output signal $S_{m}(t)$. Meanwhile, the $(a+b)$ term is the function of frequency therefore it is belongs to the phase component of signal received at $m^{\text {th }}$ antenna.

Based on this analysis, we have the phase output at each PLL is as:

$\phi_{m}=\frac{\varphi_{1}+\varphi_{2}}{2}+\frac{2 \pi \mathrm{r}}{\lambda} \cos \left(\frac{\theta_{1}-\theta_{2}}{2}\right) \cos \left(\frac{2 \pi \mathrm{m}}{M}-\frac{\theta_{1}+\theta_{2}}{2}\right)$

Define

$$
\frac{\varphi_{1}+\varphi_{2}}{2}=\varphi ; \theta=\frac{\theta_{1}+\theta_{2}}{2} ; \theta^{\prime}=\frac{\theta_{1}-\theta_{2}}{2}
$$

The Equation (28) becomes

$$
\phi_{m}=\varphi+\frac{2 \pi \mathrm{r}}{\lambda} \cos \left(\theta^{\prime}\right) \cos \left(\frac{2 \pi \mathrm{m}}{M}-\theta\right)
$$

This equation has a same form as the expected phase as given in Equation (8). As a result, the first difference curve is calculated by:

$$
\Delta \phi_{m}=-2 A \sin \left(\frac{\pi}{M}\right) \cos \left(\theta^{\prime}\right) \sin \left(\frac{2 \pi \mathrm{m}}{M}-\frac{\pi}{M}-\theta\right)
$$

where $A=2 \pi \mathrm{r} / \lambda$

Therefore we can apply the PLL-DOA algorithm with low computation complexity in this case. However, the final DOA information now becomes:

$$
\theta=\frac{\theta_{1}+\theta_{2}}{2}
$$

In order to overcome this obstacle, after finding $\theta$, we select one value in the set of true first difference values that we have calculated. Based on Equation (31), we can find the function of the difference angular between two directions, $\cos \left[\left(\theta_{1}-\theta_{2}\right) / 2\right]$, by:

$$
\begin{aligned}
B & =\cos \left(\theta^{\prime}\right) \\
& =\frac{\Delta \phi_{m}}{-2 A \sin \left(\frac{\pi}{M}\right) \sin \left(\frac{2 \pi \mathrm{m}}{M}-\frac{\pi}{M}-\theta\right)}
\end{aligned}
$$

with

$$
\theta^{\prime}=\cos ^{-1}(B)
$$

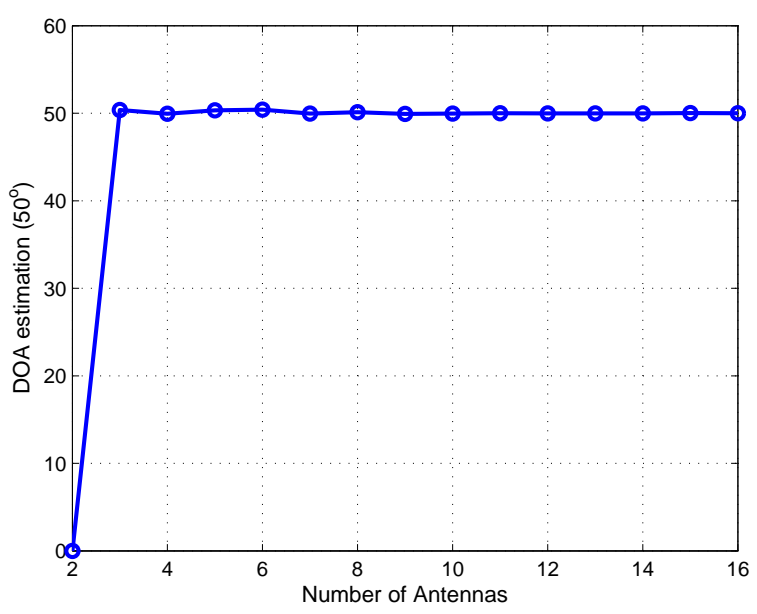

Figure 9. DOA estimation with variable number of antenna

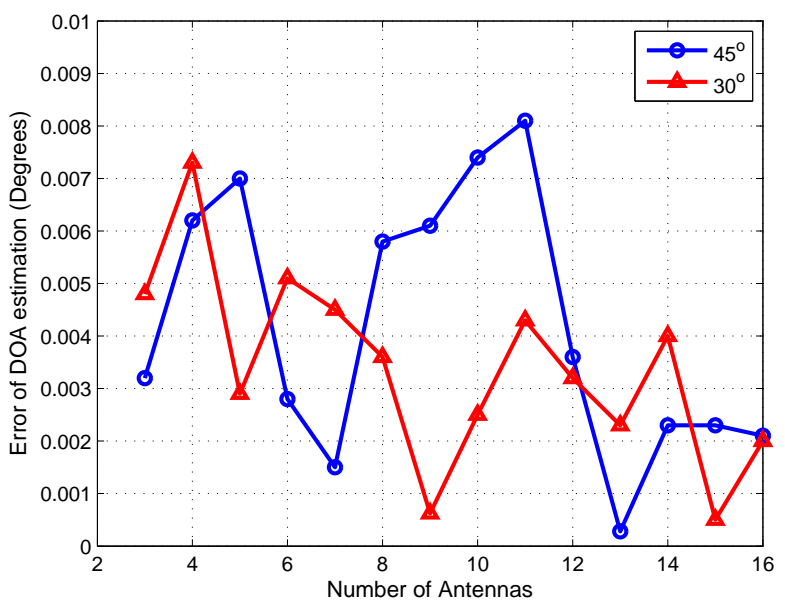

Figure 10. Error of DOA estimation (degrees) vs the number of antennas

After finding the $\theta$ and $\theta^{\prime}$, the DOAs are simply calculated by:

$$
\begin{aligned}
& \theta_{1}=\theta+\theta^{\prime} \\
& \theta_{2}=\theta-\theta^{\prime}
\end{aligned}
$$

\section{Simulation Results}

The proposed DF algorithm is simulated using Matlab in order to examine the performance of the algorithm. First we simulate our algorithm with variable number of antenna. Figure 9 shows the DOA accuracy for variable number of antenna at the DOA of 50 degrees. It can be seen that the proposed algorithm operates well when the number of antennas are more than two. However, the number of antenna elements in the array does not affect to the result accuracy significantly. To clarify this point, the errors in DOA estimations are depicted in Figure 10. In Figure 10, as we change the number of antennas, the error varies but with only small amount. It tends to decrease when the antenna's elements increase. For practical purpose, we use the model of eight elements to verify the performance of 


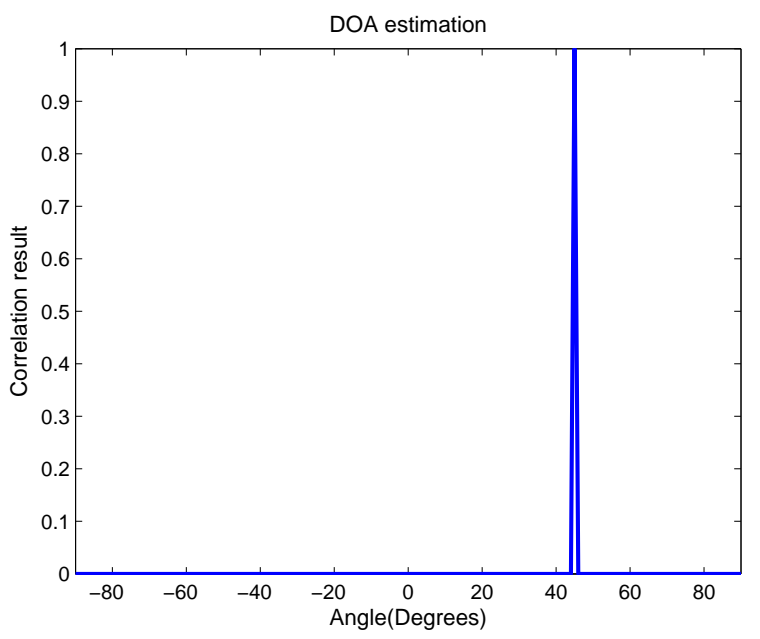

Figure 11. DOA estimation for $45^{\circ}$.

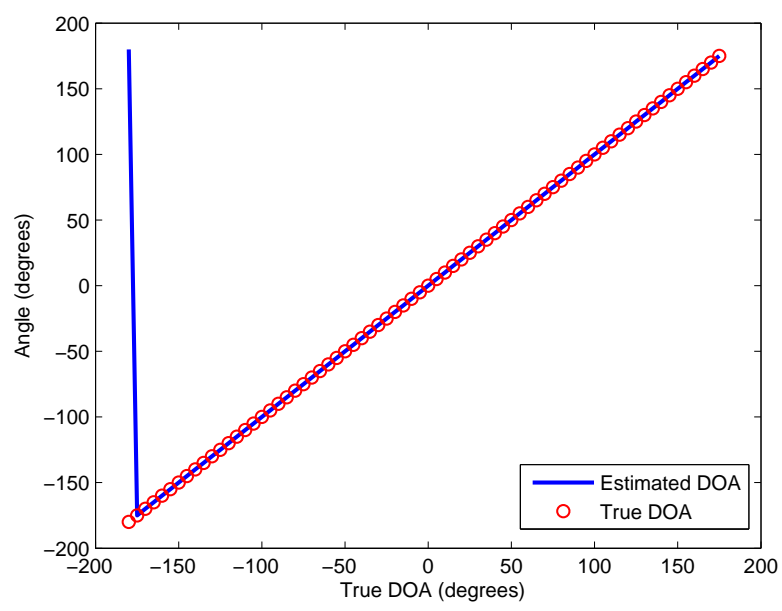

Figure 12. DOA estimation in AWGN channel.

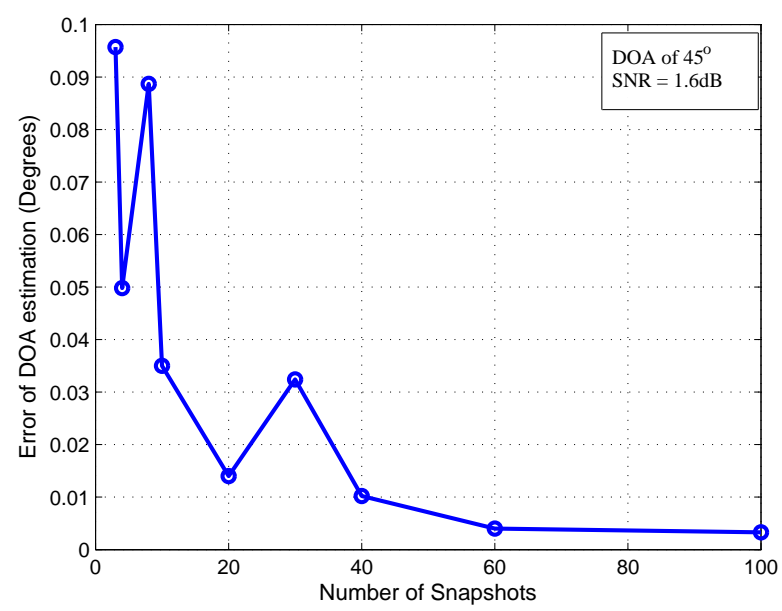

Figure 13. DOA estimation with the different number of snapshots.

the algorithm. Figure 11 plots the DOA estimation in the AWGN channel at the DOA of 45 degrees. We can see that the DOA of signal of interest is estimated accurately with the proposed algorithm with very small error.
Table I

Comparing between Convergence Time of Two Approaches

\begin{tabular}{|c|c|c|}
\hline $\begin{array}{c}\text { DOA } \\
\text { (Degrees) }\end{array}$ & $\begin{array}{c}\text { PLL - DOA algorithm } \\
\text { (Seconds) }\end{array}$ & $\begin{array}{c}\text { Proposed algorithm } \\
\text { (Seconds) }\end{array}$ \\
\hline 35 & 0.5131 & 0.0134 \\
\hline 40 & 0.5019 & 0.0131 \\
\hline 45 & 0.5103 & 0.0129 \\
\hline 53 & 0.5139 & 0.0134 \\
\hline 68 & 0.5115 & 0.0129 \\
\hline 77 & 0.5253 & 0.0132 \\
\hline 89 & 0.5505 & 0.0130 \\
\hline
\end{tabular}

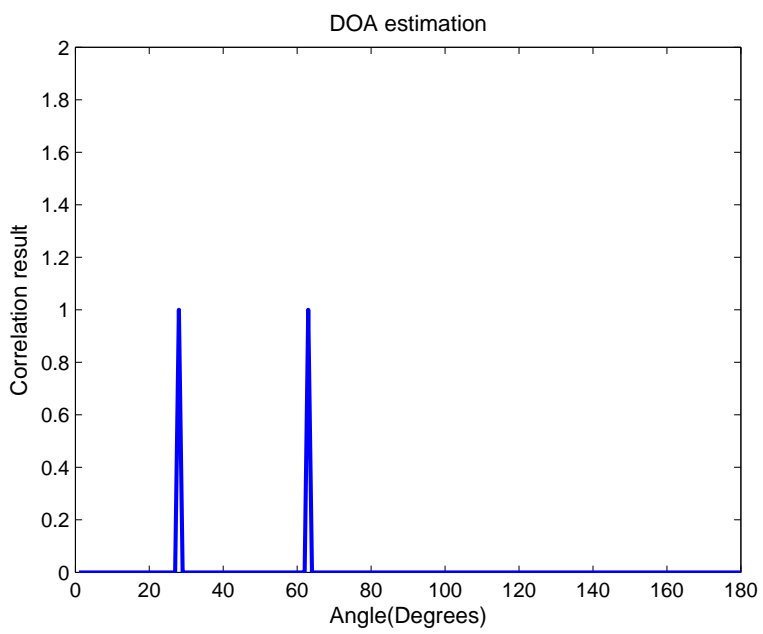

Figure 14. Simulated results for DOA estimation of 2 signals at $28^{\circ}$ and $63^{\circ}$.

In Figure 12, the results for many cases of DOAs are presented. The simulation is performed with the AWGN channel, the value $10 \mathrm{~dB}$ of SNR and 100 simulation runs per DOA. The circle shows the true DOA while the line shows the DOA estimated by our algorithm. Although at the true DOA of 180 degrees, the proposed algorithm estimates the DOA 360 degrees away, which is still correct.

Furthermore, the number of snapshots at each antenna is also a factor that makes a better estimation result. The more snapshot is taken, the better result we get. Figure 13 shows the effect of number of snapshots to DOA estimation.

In order to compare the convergence time between the PLL - DOA algorithm and the proposed method, we execute both methods with several DOAs. The results are stored in the Table.1. In the Table.1, we can easily see that the PLL - DOA algorithm with low complexity is much faster than others.

In the remainder part of our work, we verify the performance of the proposed algorithm with two incoming signals which have the same carrier frequency and equaled signal power. With this algorithm, all pairs of DOAs we tested are successfully determined. The two estimated DOA examples of 28 and 63 degrees are plotted in Figure 14.

\section{Conclusions}

We propose in this paper a new direction finding system for DOA estimation based on PLLs with low 
computation complexity. By reducing number of possible curves, the novel architecture not only has a lot of advantages of SDR receiver but also the computation complexity is significantly reduced in comparison with the conventional system. In original algorithm, the number of finding is an exponential function of the number of antenna element. With the new method, the number of finding is now only multiple of the number of antenna element. This improvement allows the possibility to reduce the time needed for computation. The proposed system with low complexity can be implemented for real time application.

\section{ACKNOWLEDGEMENTS}

This research is carried out in the framework of the project titled Design and Implementation of an Earth Station based on Software Defined Radio in the satellite communication system in the national program on space technology under the grant number VT/CN-02/14-15. This research is funded by the Vietnam Academy of Science and Technology. The authors would like to thank the Vietnam Academy of Science and Technology and the Ministry of Science and Technology, Vietnam for their financial support.

\section{REFERENCES}

[1] R. Hammerle, "Factors limiting the accuracy of doppler and adcock direction finding systems," in Proc. of 1989 IEE Colloquium on Passive Direction Finding, Jan. 1989, pp. $3 / 1-3 / 13$.

[2] R. Products, "A comparison of the watson-watt and pseudo-doppler df techniques," in Web note WN-004, April 1999, pp. Rev. B-01.

[3] N. Harter, J. J. Keaveny, S. Venkatesh, and R. M. Buehrer, "Analysis and implementation of a novel single-channel direction finding algorithm," in Proc. Of 2005 IEEE Wireless Communications and Networking Conference, vol. 4, March 2005, pp. 2530-2533.

[4] S. V. N. Harter, J. J. Keaveny and R. M. Buehrer, “Analysis and implementation of a novel single-channel direction finding algorithm," in DRS Signal Solutions Technical Symposium, May 2005.

[5] N. Harter, J. J. Keaveny, S. Venkatesh, and R. M. Buehrer, "Development of a novel single-channel direction finding algorithm," in Proc. Of 2005 IEEE Military Communications Conference, vol. 4, October 2005, pp. 2720-2725.

[6] S. F. Cotter, "Multiple snapshot matching pursuit for direction of arrival (doa) estimation," in 15th European Signal Processing Conference, EUSIPCO, Virginia, 2007.

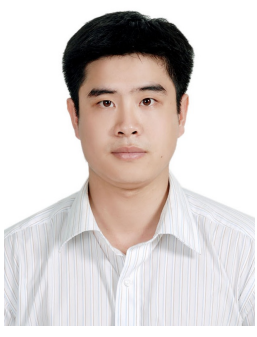

tenna array.
Han Trong Thanh was born in Ha Noi, Vietnam, in 1985. He received the Dipl. of Engineer in 2008 and Master of Science in 2010 from the School of Electronics and Telecommunications, Hanoi University of Science and Technology, Vietnam. Currently, he is a Ph.D candidate at Dept. of Telecommunication Systems, School of Electronics and Telecommunications, Hanoi University of Science and Technology, Vietnam. His research interests are localization and signal processing for an-

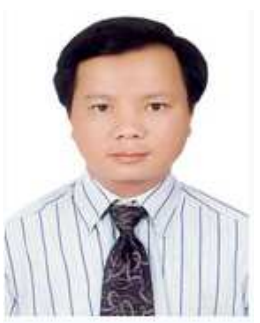

Vu Van Yem was born in 1975 in Hai Phong, Vietnam. He received the Ph.D. degree in communications from Department of Electronics and Communications, TELECOM ParisTech (formerly ENST Paris) France in 2005. From 2006 to 2007, he was a postdoctoral researcher at the Department of Hyper-frequencies and Semiconductor, Institute of Electronics, Microelectronics and Nanotechnology (IEMN), France. He has been qualified to be named as Associate Professor since November, 2009. Currently, he is the Deputy - Dean of Graduate School and the Head of the Department of Telecommunication Systems, School of Electronics and Telecommunications, Hanoi University of Science and Technology, Vietnam. His area of expertise are microwave engineering, antenna, chaos-based digital communications as well as wireless communication and localization systems. 